\title{
Source Camera Identification with Imbalanced Training Dataset
}

\author{
Yonggang Huang ${ }^{1, *}$, Jun Zhang ${ }^{2}$, Xinkai Lan ${ }^{3}$ \\ ${ }^{1}$ School of Computer Science and Technology, Beijing Institute of Technology, \\ Beijing, 100081, China \\ ${ }^{2}$ School of Information Technology, Deakin University, VIC, 3217, Australia \\ ${ }^{3}$ Overhaul Branch, Beijing Electric Power Company, Beijing, China \\ E-mail: yonggang.h@gmail.com
}

\begin{abstract}
In this paper, we address the problem of unbalanced training dataset for source camera identification, namely, there are fewer training examples for some camera models compared to other camera models. A new source camera identification approach is proposed to alleviate the influence of imbalanced training dataset. In the proposed approach, firstly, we treat source camera identification as a multi-class classification problem, and decompose it into binary classification problems. After decomposing, the problem of imbalanced training dataset for multiclass classification is transformed to the problem of imbalanced training dataset for binary classification. Then, we incorporate SMOTE and AdaBoost algorithms to construct SVM ensemble to address the issue of imbalanced training dataset for binary classification. A number of experiments show the proposed approach can deal with the imbalanced training dataset effectively.
\end{abstract}

Keywords: Source camera identification; unbalanced training dataset; SVM ensemble.

\section{Introduction}

With the development of digital imaging technologies and the popularity of digital cameras, digital images have become increasingly prevalent in today's daily life. The digital images are used as photographic evidence to make critical decisions by many governmental, legal, scientific, and news media organizations [1]. On the other side, with the widespread availability of image editing software, the creation and manipulation of digital images become easy for deceiving others from the truth. In such a scenario, image authentication and image integrity validation are challenging when the images are used as evidences. Image forensics is an emerging field concerned with the two issues [2]. Image authentication is to identify the source imaging device of an image. Image integrity validation is to determine whether and how a digital image has been manipulated. In this paper, we focus on image authentication, i.e. identifying the source camera of a given image.

Source camera identification has many applications. For example, verifying the original source of evidence images in court, finding the rightful owner of images in the case of copyright dispute [2], and etc. A simple approach for source camera identification is to use the image header, since the image source related information such as camera brand, model, date, and time are embedded in the header [3]. However, the image header is very easy to manipulate and unreliable in practice. Studies on watermark $[4,5]$ try to embed in the image an invisible or visible watermark, which carries the source related information such as camera brand, model and time. It seems that inserting watermark directly into the image is an elegant solution to the source camera identification. However, watermarks need to be inserted during the creation of an image. This complicates the design and 
increases the production cost of digital camera. In fact, most images captured today do not contain digital watermarks. The absence of widespread adoption of digital watermarks makes it hard to clarify the source of images.

Usually the process of acquiring an image with a digital camera is as follows [2, 6]: the light from the scenes passes through camera lens, anti-aliasing filter, and color filter array (CFA); then the following semiconductor sensor converts the light into electronic signal; finally post-processing operations including gamma correction, demosaicking, image correction, white balance and JPEG compression are performed before the image is saved in the storage. The hardware and software in the image acquisition process may leave traces in images. These unique traces can be used to identify the imaging camera. Compared to the image header and watermark based approaches, source camera identification relying on the image acquisition process is much practical.

Image acquisition process driven approaches firstly extract features on intrinsic hardware artifacts or software-related fingerprints left during the image acquisition process, and then cast the identification as a classification problem [7, 8]. Hardware imperfections includes pattern noise [6, 9, 10], lens radial distortion [11, 12], chromatic aberration [13], sensor dust [14, 15] and etc. Software-related fingerprints include image-related features [8], artifacts introduced by color filter array [7] and etc. Based on the features extracted from the images, supervised classifiers are built to perform source camera identification. Although there exists a large variety of various machine learning tools, support vector machine (SVM) [16] seems to be the most popular choice. This is because SVMs are resistant to overtraining and perform very well even when the feature dimensionality is comparable or larger than the size of the training dataset. Image acquisition process driven approaches firstly extract features on intrinsic hardware artifacts or software-related fingerprints left during the image acquisition process, and then cast the identification as a classification problem [2, 23]. Hardware imperfections includes pattern noise [20, 22, 28], lens radial distortion [25, 26], chromatic aberration [31], sensor dust [11, 12] and etc. Software-related fingerprints include image-related features [23], artifacts introduced by color filter array [2] and etc. Based on the features extracted from the images, supervised classifiers are built to perform source camera identification. Although there exists a large variety of various machine learning tools, support vector machine (SVM) [8] seems to be the most popular choice. This is because SVMs are resistant to overtraining and perform very well even when the feature dimensionality is comparable or larger than the size of the training dataset.

However, SVM classifier based source camera identification methods suffer from poor performance when the training dataset have imbalanced class distribution. That is, there are fewer training examples for some camera models compared to other camera models. The SVM algorithm works well with balanced training datasets. With imbalanced training datasets, the decision boundary produced by SVM tends to be biased towards the majority classes, and the instances from minority classes are likely to be misclassified. Consequently, the images from the camera model with fewer training examples will be inaccurately identified from the camera model with more training examples.

We aim to tackle the problem of imbalanced training dataset for source camera identification, and a new source camera identification approach is proposed in this paper. We propose a new SVM ensemble method to address the new problem of imbalanced training dataset for source camera identification. Firstly, we treat source camera identification as a multi-class classification problem, and decompose it into binary classification problems. After decomposing, the problem of imbalanced training dataset for multi-class classification is transformed to the problem of 
imbalanced training dataset for binary classification. Then, we incorporate SMOTE [17] and AdaBoost [18] algorithms to construct SVM ensemble to address the issue of imbalanced training dataset for the binary classification.

The remainder of the paper is structured as follows. Some related work is briefly reviewed in Section II. Section III presents the new source camera identification approach in detail. Section IV presents the experimental results. Finally, the paper is concluded in Section V.

\section{Related Work}

\subsection{Image Acquisition Process Driven Source Camera Identification}

Image acquisition process driven approaches firstly extract features on intrinsic hardware artifacts or on software-related fingerprints left during the image acquisition process, and then cast the identification as a supervised classification problem. A lot of researches have been devoted to design sophisticated features which can character the traces left during image acquisition. Kharrazi et al. [8] believed that an output image is greatly effected by CFA configuration/demosaicing algorithm and color processing/transformation. They proposed a total of 34 features to capture the differences in the underlying color characteristics for different cameras. Geradts et al. [19] showed that hot pixels or dead pixels (defective pixels in general), could be used for reliable camera identification even for lossy JPEG compressed images. Luk'a ss et al. [9] proposed a method for the problem of source camera identification based on the pixel nonuniformity noise, which is a stochastic component of the pattern noise common to all digital imaging sensors. Dirik et al. [14] believed that the location and shape of dust specks in front of the imaging sensor and their persistence make dust spots a useful fingerprint for digital single lens reflex cameras. They proposed an approach based on sensor dust traces for source camera identification. Choi et al. [11] performed source camera identification using intrinsic lens radial distortion. The underlying idea is that the majority of digital cameras are equipped with lenses having spherical surfaces, whose inherent radial distortions serve as unique fingerprints in the images. The authors extracted parameters from aberration measurements for the source camera identification task. Van et al. [13] observed the chromatic aberration phenomenon, where lights of different wavelengths fail to converge at the same position on the focal plane. They estimated the parameters of lateral chromatic aberration and used them as features for source camera identification.

Existed research efforts focus on developing sophisticated features for source camera identification. On the other hand, the problems of imbalanced training dataset have not been addressed.

\subsection{SVM Ensemble for Imbalanced Datasets}

The SVM is a promising classification and regression algorithm proposed by Cortes and Vapnik [16]. The SVM learns a separating hyperplane to maximize the margin and to produce a good generalization capability. SVMs have gained success in many applications, such as image retrieval [20], text mining [21], hand-writing recognition [22] and speaker recognition [31]. However, when the data is highly imbalanced, the decision boundary obtained from the training dataset is biased toward the minority class.

In machine learning, classifier ensemble [23] is an effective approach to improve the classification accuracy by inducing several classifiers and combining them to obtain a new classifier. Recently, SVM ensemble has arisen as a possible solution to 
the imbalanced training datasets [24-26]. These researches usually embed data processing technologies (typically the data sampling technologies) in the SVM ensemble construction process. For example, Yan et al. [24] proposed to use SVM ensemble to address the issue of imbalanced training dataset. They firstly decomposed majority class into partitions, and combined the minority class with each partition of majority class to be an individual subset. Then they trained SVMs independently on every subset of the training dataset, and finally combined all constituent SVMs. Kang et al. [25] proposed an Ensemble of Under-Sampled SVMs (EUS SVMs) for the problem of imbalanced training datasets. EUS SVMs build multiple different training sets by under-sampling examples from the majority class and combing them with the minority class examples. Then, each training set is used for training an individual SVM classifier. Finally, the output of the ensemble is produced by aggregating the outputs of all individual classifiers. Liu et al. [26] proposed a new SVM method called EnSVM, Different from previous researches $[24,25]$, EnSVM integrates oversampling and undersampling together in the SVM ensemble approach. That is, EnSVM first over-samples the minority class to some extent, and then under-samples the majority class to make both sides have the same size.

SVMs were originally designed for binary classification, and it can be extended for multi-class classification task [27]. Compared to binary classification, multiclass classification is much complicated. Moreover, the imbalance of training dataset may increase the difficulty of multi-class classification. Existed researches [24-26] focus on binary-class imbalance problem, and the proposed SVM ensemble solutions can not be directly applied for the multi-class imbalance problem.

\section{A New Source Camera Identification Approach}

In this section, we present a novel source camera identification approach with imbalanced training dataset.

\subsection{System Model}

Fig. 1 shows a novel system model which can alleviate the influence of imbalanced training dataset. In Fig.1, the blue solid arrows indicate the data flow of the training stage for source camera identification, and the red solid arrows indicate the data flow of the classifying (predicting) stage. At the training stage, firstly, features on intrinsic hardware artifacts or on software-related fingerprints are extracted from the images. Then, the SVM ensembles are constructed and trained. At the predicting stage, firstly, features are extracted from the input images for identification. Then, the features are input into the trained SVM ensembles, and the predicted results of the SVMs are aggregated. 


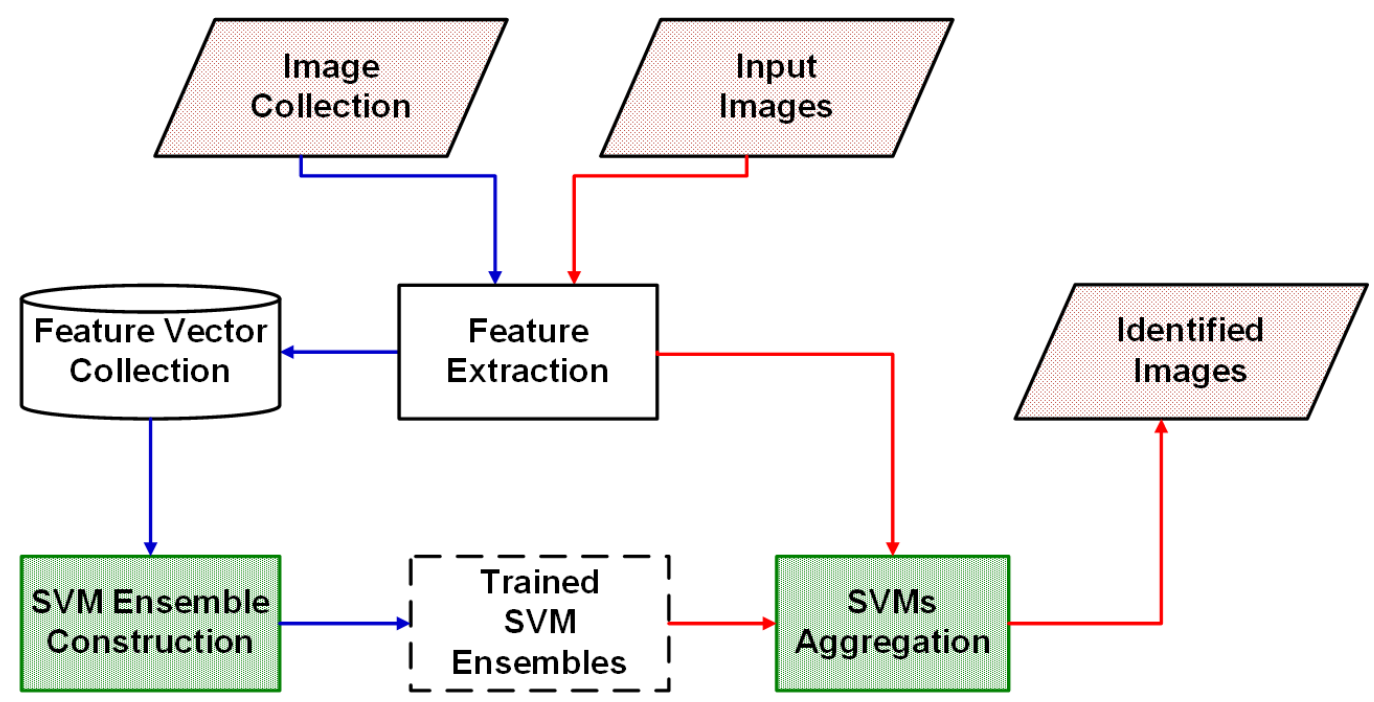

Figure 1. The System Model

\subsection{The Proposed Approach}

Since there are multiple camera models, the source camera identification can be regarded as a multiclass classification problem. The boundaries among the classes can be overlapped, which makes the classification much complicated. Moreover, the imbalanced training dataset may increase the difficulty of multi-class classification. In this situation, we decompose the original multi-class classification problem into binary classification problems. We make use of the One-Against-One [27] approach to decompose the multi-class classification problem, which constructs $\mathrm{k}(\mathrm{k}-1) / 2$ binary classifiers for a k-class classification task. One binary classifier is trained for each possible class pair. After decomposing, the problem of imbalanced training dataset for multi-class classification is transformed to the problem of imbalanced training dataset for binary classification.

SVM ensemble have been applied to address the issue of imbalanced training dataset in recent researchesand gained positive results [24-26]. In this paper, we propose a new SVM ensemblemethod by combing SMOTE and AdaBoost algorithms.

- Firstly, we oversample the minority class using SMOTE to a moderate extent. In SMOTE, randomsynthetic minority instances are introduced along the line segments connecting a minority instance and its neighbours [17].

- Then, we use the AdaBoost [18] to construct the SVM ensemble on the oversampled minority class and the majority class. The AdaBoost algorithm creates a set of base classifiers iteratively by adaptively adjusting the weights over examples in the training dataset. During each iteration, the misclassified instances in current classifier are given higher weights, and vice versa.

The detailed process for SVM ensemble construction is described in Algorithm 1.

The proposed SVM ensemble method alleviates the influence of imbalanced training dataset in three aspects. Firstly, oversampling with SMOTE reduces the level of imbalance. Secondly, during each iteration of AdaBoost, the minority class examples tend to be given higher weights since they are most likely to be misclassified. In this way, the influence of imbalanced training dataset is further 
alleviated. Thirdly, SVM ensemble can overcome the unstabiblity of a single SVM built on imbalanced training dataset and achieve better performance.

Finally, the outputs of SVMs are aggregated using majority voting method.

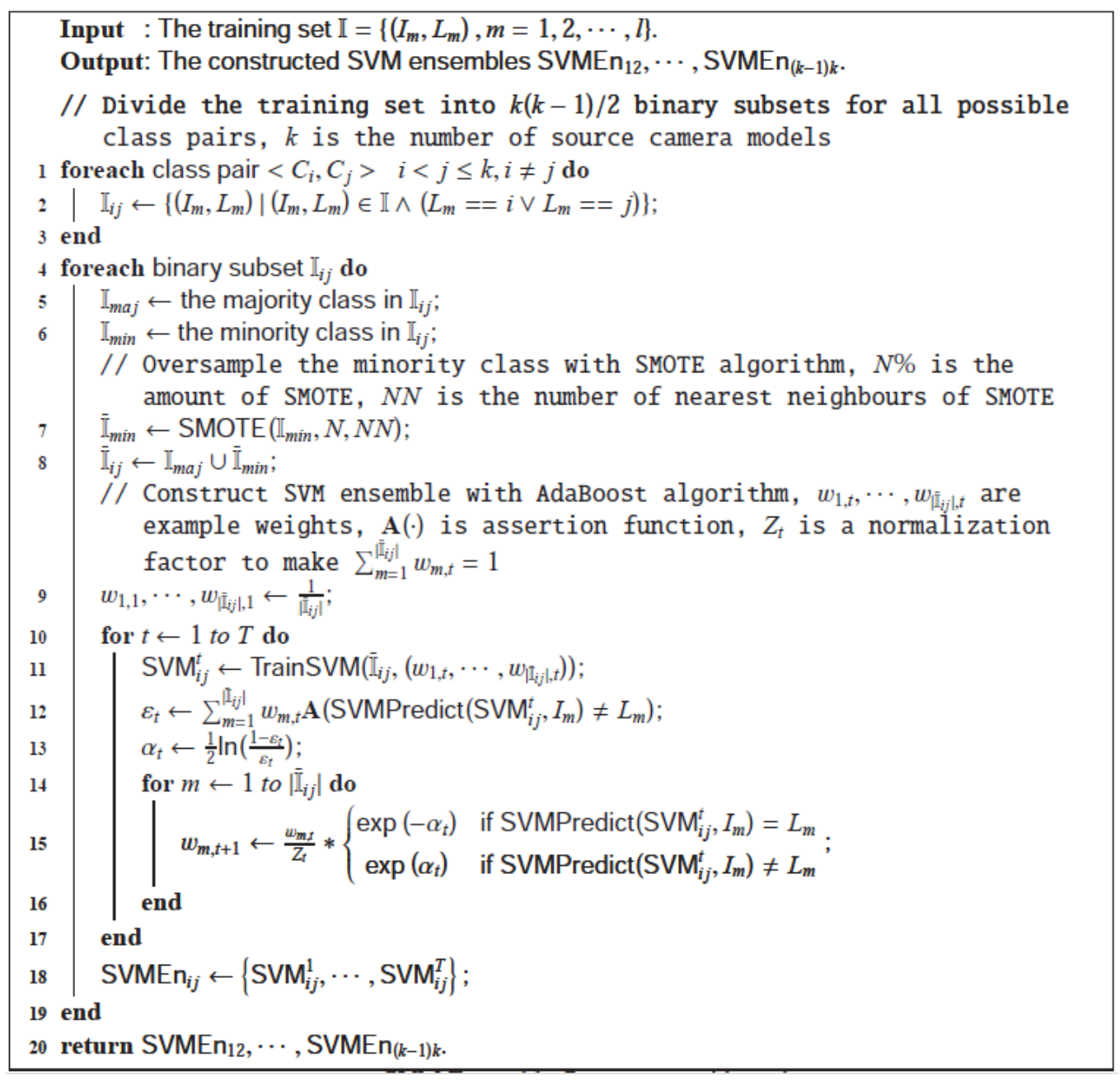

Algorithm 1. SVM Ensemble Construction Algorithm

\section{Experiments and Results}

To evaluate the proposed scheme, we performed a number of experiments on the Dresden image collection [28]. The image database is specifically built for the purpose of development and benchmarking of camera-based digital forensic techniques. In order to simulate a realistic scenario of imbalanced training dataset, 7 camera models with varied image set size were selected. The details of the selected camera models is present in Table.1. 
Table 1. The Details of the Selected Camera Models

\begin{tabular}{|c|c|c|c|c|}
\hline No. & Camera Model & Alias & Training Size & Test Size \\
\hline 1 & Agfa_DC $-830 i$ & A1 & 33 & 330 \\
\hline 2 & Agfa_Sensor530s & A2 & 42 & 330 \\
\hline 3 & Kodak_M1063 & K & 2061 & 330 \\
\hline 4 & Nikon_D200 & N & 422 & 330 \\
\hline 5 & Olympus_mju_1050SW & O & 710 & 330 \\
\hline 6 & Panasonic_DMC - FZ50 & P1 & 601 & 330 \\
\hline 7 & Pentax_OptioA40 & P2 & 308 & 330 \\
\hline
\end{tabular}

We extracted 34 features proposed by Kharrazi et al. [8] to character the images. Those 34 features includes: average pixel value ( 3 features), RGB pairs correlation (3 features), neighbor distribution center of mass (3 features), RGB pairs energy ratio ( 3 features), wavelet domain statistics ( 9 features) and image quality metrics (13 features).

We used the LIBSVM [29] to solve SVMs. Linear kernels were applied in our experiments.

The state of art SVM ensemble method for imbalanced training dataset proposed in [26], namely EnSVM, was implemented as reference. In addition, in order to evaluate the contributions of SMOTE and AdaBoost in the proposed method respectively, the two algorithms were also implemented as references. The majority voting aggregation method was used to aggregate the outputs of SVMs.

Fig.2 and Fig.3 shows the identification accuracy and F-Measure of the five comparative methods, respectively. We can see that the proposed method outperforms EnSVM. The reason is that the AdaBoost algorithm used in the proposed method is more effective at dealing with the imbalanced training dataset than the bagging algorithm [30], which is adopted in EnSVM. The figures also show that both SMOTE and AdaBoost achieve better performance than the original method. This indicates that the two algorithms contribute to the overall performance of the proposed SVM ensemble method.

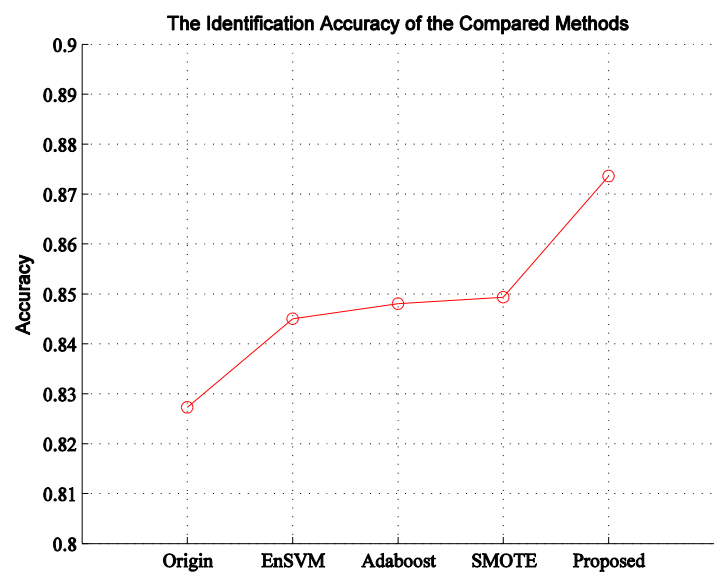

Figure 2. The Identification Accuracy of the Compared Methods 


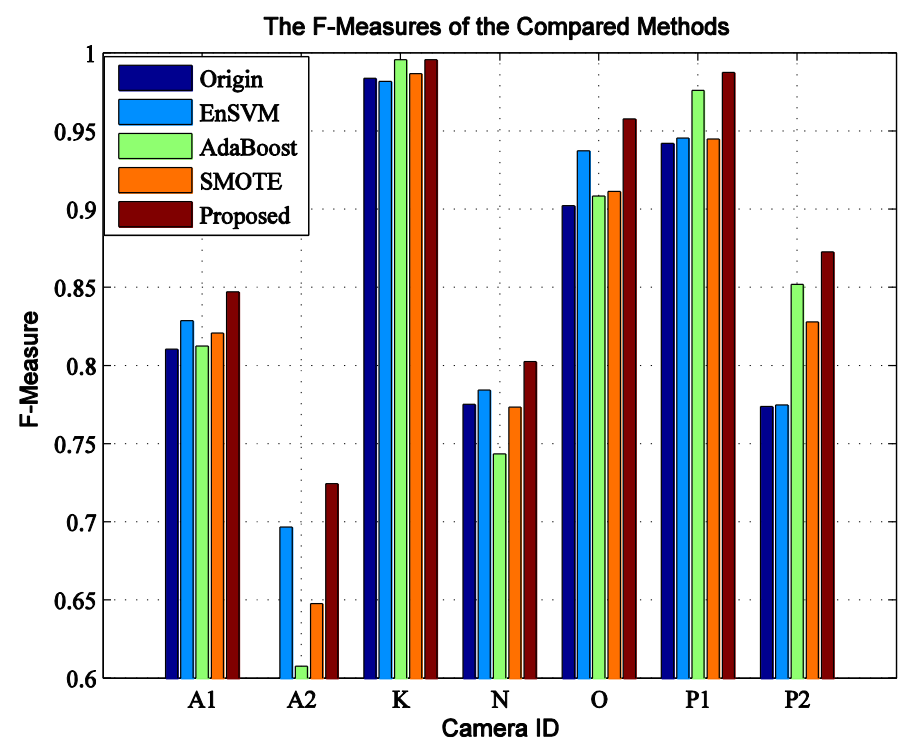

Figure 3. The F-Measures of the Compared Methods

\section{Conclusion}

Traditional source camera identification scheme suffers from poor performance when the training data have imbalanced class distribution. In this paper, a new source camera approach is proposed to address this issue. In the proposed approach, firstly, we treat source camera identification as a multi-class classification problem, and decompose it into binary classification problems. After decomposing, the problem of imbalanced training dataset for multi-class classification is transformed to the problem of imbalanced training dataset for binary classification. Then, we incorporate SMOTE and AdaBoost algorithms to construct SVM ensemble to address the issue of imbalanced training dataset for binary classification. A number of experiments carried out on a real-world image collection, and the experimental results demonstrated the proposed source camera identification can effectively alleviate the influence the imbalanced training dataset.

\section{Acknowledgement}

This work is supported by the National Natural Science Foundation of China (No. 61300077), the National 863 Programme (No. 2013AA01A212) and the Basic Research Foundation of Beijing Institute of Technology (No. 20130742004).

\section{References}

[1] M. C. Stamm and K. J. R. Liu, "Forensic detection of image manipulation using statistical intrinsic fingerprints," IEEE Transactions on Information Forensics and Security, vol. 5, no. 3, (2010), pp. 492506.

[2] Z. Deng, A. Gijsenij and J. Zhang, "Source camera identification using auto-white balance approximation," in The 13th IEEE International Conference on Computer Vision, Barcelona, Spain, (2011).

[3] O. C. Eliktutan, B. Sankur and I. Avcibas,, "Blind identification of source cell-phone model", IEEE Transactions on Information Forensics and Security, vol. 3, no. 3, (2008), pp. 553-566.

[4] I. J. Cox, M. L. Miller and J. A. Bloom, "Digital watermarking. San Francisco", CA, USA: Morgan Kaufmann Publishers Inc., (2002). 
[5] R. Schyndel, A. Z. Tirkel and C. F. Osborne, "A digital watermark", in The 1st IEEE International Conference Image Processing, vol. 2, Austin, Texas, USA, (1994).

[6] C. T. Li, "Source camera identification using enhanced sensor pattern noise", IEEE Transactions on Information Forensics and Security, vol. 5, no. 2, (2010), pp. 280-287.

[7] S. Bayram, H. Sencar, N. Memon and I. Avcibas, "Source camera identification based on CFA interpolation", in The 12th IEEE International Conference on Image Processing, Genoa, Italy, vol. 3, (2005).

[8] K. Mehdi, H. Sencar and N. Memon, "Blind source camera identification", in The 11th IEEE International Conference on Image Processing, Singapore, (2004).

[9] J. Luk'a s, J. Fridrich and M. Goljan, "Digital camera identification from sensor pattern noise", IEEE Transactions on Information Forensics and Security, vol. 1, no. 2, (2006), pp. 205-214.

[10] Y. Sutcu, S. Bayram, H. T. Sencar and N. Memon, "Improvements on sensor noise based source camera identification", in The 2007 IEEE International Conference on Multimedia and Expo, Beijing, China, (2007).

[11] K. S. Choi, E. Y. Lam and K. K. Wong, "Automatic source camera identification using the intrinsic lens radial distortion”, Optics express, vol. 14, no. 24, (2006), pp. 11 551-11 565.

[12] K. S. Choi and E. Y. Lam, "Source camera identification using footprints from lens aberration", in Proceeding of SPIE-IS and Electronic Imaging, (2006).

[13] L. T. Van, S. Emmanuel and M. S. Kankanhalli, "Identifying source cell phone using chromatic aberration", The 2007 IEEE International Conference on Multimedia and Expo, Beijing, China, (2007), pp. $883-886$.

[14] A. E. Dirik, H. T. Sencar and N. Memon, "Digital single lens reflex camera identification from traces of sensor dust", IEEE Transactions on Information Forensics and Security, vol. 3, no. 3, (2008), pp. 539552.

[15] A. E. Dirik and H. T. Sencar, "Source camera identification based on sensor dust characteristics", in The 2007 IEEE Workshop on Signal Processing Applications for Public Security and Forensics, Washington, DC, USA, (2007).

[16] C. Cortes and V. Vapnik, "Support-vector networks", Machine learning, vol. 20, no. 3, (1995), pp. 273 297.

[17] N. V. Chawla, K. W. Bowyer, L. O. Hall and W. P. Kegelmeyer, "SMOTE: synthetic minority oversampling technique”, Journal of Artificial Intelligence Research, vol. 16, no. 1, (2002), pp. 321-357.

[18] Y. Freund and R. E Schapire, "A decision-theoretic generalization of on-line learning and an application to boosting", Journal of Computer and System Sciences, vol. 55, no. 1, (1997), pp. 119-139.

[19] Z. J. Geradts, J. Bijhold, M. Kieft, K. Kurosawa, K. Kuroki and N. Saitoh, "Methods for identification of images acquired with digital cameras", in Proceeding SPIE 4232, Enabling Technologies for Law Enforcement, (2001).

[20] D. Tao, X. Tang, X. Li and X. Wu, "Asymmetric bagging and random subspace for support vector machines-based relevance feedback in image retrieval", IEEE Transactions on Pattern Analysis and Machine Intelligence, vol. 28, no. 7, (2006), pp. 1088-1099.

[21] S. Tong and D. Koller, "Support vector machine active learning with applications to text classification", Journal of Machine Learning Research, vol. 2, no. 1, (2001), pp. 45-66.

[22] C. Bahlmann, B. Haasdonk and H. Burkhardt, "Online handwriting recognition with support vector machines - a kernel approach", in The 8th International Workshop on Frontiers in Handwriting Recognition, Ontario, Canada, (2002).

[23] T. G. Dietterich, "Ensemble methods in machine learning", in Multiple Classifier Systems. Springer Berlin Heidelberg, (2000).

[24] R. Yan, Y. Liu, R. Jin and H. Alex, "On predicting rare classes with svm ensembles in scene classification", in The 2003 IEEE International Conference on Acoustics, Speech, and Signal Processing, vol. 3, Hong Kong, China, (2003).

[25] P. Kang and S. Cho, "EUS SVMs: Ensemble of under-sampled SVMs for data imbalance problems", in The13th International Conference Neural Information Processing, Hong Kong, China, (2006).

[26] Y. Liu, X. Yu, J. X. Huang and A. An, "Combining integrated sampling with svm ensembles for learning from imbalanced datasets", Information Processing and Management, vol. 47, no. 4, (2011), pp. $617-631$.

[27] C. W. Hsu and C. J. Lin, "A comparison of methods for multiclass support vector machines", IEEE Transactions on Neural Networks, vol. 13, no. 2, (2002), pp. 415-425.

[28] T. Gloe and R. B"ohme, "The dresden image database for benchmarking digital image forensics", Journal of Digital Forensic Practice, vol. 3, no. 2-4, (2010), pp. 150-159.

[29] C. Chang and C. Lin, "LIBSVM: a library for support vector machines", National Taiwan University, http://www.csie.ntu.edu.tw/ cjlin/libsvm/, accessed, (2013).

[30] L. Breiman, "Bagging predictors", Machine learning, vol. 24, no. 2, (1996), pp. 123-140.

[31] Z. Y. Gao, Y. S. Zhang and M. K. Wang, "Speaker Recognition Using Kernel K-mean Clustering and SVM", Journal of Harbin University of Science and Technology, vo1. 12, no. 12, (2008), pp. 40-42. 
International Journal of Database and Theory and Application Vol.9, No.2 (2016) 